\title{
Evidence Based Medicine: from Science to Patient
}

\section{Thomas Scholz*}

Aesthetic \& Plastic Surgery Institute, University of California, Irvine, 200 S. Manchester Avenue, Suite 650, Orange, CA 92868-3298, USA

Is evidence based medicine always the primary aim in patient care? Considering that medicine is almost as old as human mankind, the advent of evidence based concepts to improve our medical knowledge and treatments is a fairly recent development. The idea of evidence based medicine dates back to the second half of the eighteenth century. At that time, British doctors developed the concept of "medical arithmetic" suggesting that medical analyses of disease processes would allow for progress and improvement of patient treatment. British doctors published the first controlled clinical studies as early as 1753 investigating therapeutic options in the treatment of scurvy. However, it took until 1793 for the term "evidence based medicine" to be described in a publication by the Scottish doctor George Fordyce [1]. Several decades later the "systematic clinical observation" was popular all over continental Europe. The foundation of the current endeavor and the new era of evidence based medicine was set by the British epidemiologist Archie Cochrane. His book "Effectiveness and Efficiency: Random Reflections on Health Services"-published in 1972-marks this beginning and outlines the modern perspectives on evidence based medicine [2].

Evidence based medicine is defined as "the conscientious, explicit, and judicious use of current best evidence in making decisions about the care of individual patients". Therefore, the practice of evidence based medicine is about the decision making process for with regards to an individual patient. This decision making process integrates two areas of expertise: the "current best evidence" and the individual clinical experience. The current best evidence means all clinically relevant systematic research in one defined area. This provides medical facts, gives power to diagnostic tests, safety, efficacy, and confidence in the treatment of disease processes. Individual clinical experience is less clearly defined, but means the individual expertise of a doctor with all the qualities that he acquired in regards to patient care. A good example for these two main areas of the practice of evidence based medicine is the job as a surgeon. This example demonstrates that by performing technical procedures, a surgeon cannot solely rely on current best evidence. He needs all his expertise, his experience and his technical skills to perform surgeries in order to make a difference in the patient's life. Every good doctor should try to use both when engaged in patient care, the current best evidence and his own clinical expertise. Both are needed, neither one can stand alone. If we only rely on the experience of a doctor, standard of care will be out of date rapidly and never be able to improve significantly. If we only rely on the current best evidence, our standard of care may be inappropriate or even detrimental to the individual patient. In short, if you rely on experience only or on systematic research only, it is not evidence based medicine. But how much medicine is evidence based? The website of the British Medical Journal demonstrates that today $51 \%$ of medicine is of unknown effectiveness. Furthermore, $3 \%$ are likely to be ineffective or harmful, $5 \%$ unlikely to be beneficial, $7 \%$ trade-off between benefits and harm, $23 \%$ likely to be beneficial and only $11 \%$ beneficial.

Different systems have been created to define certain levels of evidence and to give a systematic hierarchy to research. Several official institutions that are setting the standards and give recommendations on each level of evidence include the Cochrane collaboration [3], the
Oxford Center of Evidence Based Medicine [4], and the GRADE group [5], among others.

The question remains: what are we doing with evidence based medicine and what does it mean for the patient? One main focus of evidence based medicine is to close the gap between science and patient. Today, some applications make it faster to go from the bench to the bedside than others. Those applications are mostly more attractive, simple, or inexpensive. Viral transfections in tissue engineering are well established, but a viral treatment would probably never be very attractive to any patient. In contrast, cell products from umbilical cords or placentas are already in clinical use for wound healing purposes. Evidence based medicine ensures that basic science research meets the clinician's demands and experiences and finds its way to the patient. The guidelines for these processes are set and supervised by each government to provide safety and effectiveness to each new development. This week Lancet published a multicenter study including 28 European countries, demonstrating that the mortality after general surgery is $4 \%$ which is more than double than previous estimates. Additionally mortality rates vary widely between the countries observed [6]. Outcome studies such as these dictate the need for improvement in health care. New systematic research together with the expertise of European clinicians will work on improving these outcomes.

The most important link between basic science and patients are clinicians who keep up with medical advances. They are the front line and closest in closing the gap. They are busy doctors that spend their scarce time reading and dedicating themselves to research thus understanding both areas. As mentioned above, "current best evidence" cannot replace individual clinical expertise and evidence based medicine cannot exist without clinical experience. This is very important to understand when trying to create or change health care systems. That's why it is paramount that people responsible for health care systems are not just armchair warriors dedicated only to cost cutting thus misusing evidence base medicine. It requires experienced and knowledgeable politicians who understand the real meaning of evidence based medicine and know how to utilize it to create an advantage for society with all its social responsibilities. It further requires listening to clinicians dedicated to evidence based medicine as they are the most important link in the chain of bringing new findings appropriately to the patient. The positive impacts of evidence based medicine have just begun to be beneficial for society and will continue

*Corresponding author: Thomas Scholz, Aesthetic \& Plastic Surgery Institute University of California, Irvine, $200 \mathrm{~S}$. Manchester Avenue, Suite 650 Orange, CA 92868-3298, USA, Tel: (714) 456-5253; Fax: (714) 456-7718; E-mail: tscholz@uci.edu

Received September 27, 2012; Accepted September 28, 2012; Published October 01, 2012

Citation: Scholz T (2012) Evidence Based Medicine: from Science to Patient. J Tissue Sci Eng 3:e113. doi:10.4172/2157-7552.1000e113

Copyright: (c) 2012 Scholz T. This is an open-access article distributed under the terms of the Creative Commons Attribution License, which permits unrestricted use, distribution, and reproduction in any medium, provided the original author and source are credited. 
to evolve to become an integral part and important factor for every future health care system.

Finally, bringing science to everyday clinical practice is more likely to develop in a favorable environment where scientists and clinicians work closely together. There is a wide spectrum of new approaches, inventions, and treatments that have been developed through bench work that never reached clinical studies or beyond. These are ideas which work well, but have never been approved and are sitting on a shelf somewhere in some research building. On the other hand clinicians often times perform a treatment knowing that it will work and help the individual patient, but without understanding the precise mechanisms behind it or why it works.

Is evidence based medicine always the primary aim in patient care? I cannot answer this question, but as a clinician I can say that evidence based medicine helps me be a better physician and provide better care for my patients. Being part of a basic science idea from initial brainstorming via the different phases of translational research and clinical analysis to definite application in patient care is a fascinating and rewarding process. Therefore, it is the evidence based medicine that not only brings magic and passion to scientists and clinicians, but even more so the hope and the promise on the horizon that it may be able to change so many people's lives in the future.

\section{References}

1. Fordyce G (1793) An attempt to improve the evidence of medicine. Transactions of a Society for the Improvement of Medical and Chirurgical Knowledge. J Johnson, London, UK.

2. Cochrane AL (1972) Effectiveness and efficiency: random reflections on health services. (1stedn), Nuffield Provincial Hospitals Trust, London, UK.

3. http://www.cochrane.org

4. Oxford Centre for Evidence-Based Medicine Levels of Evidence Working Group.

5. Guyatt GH, Oxman AD, Vist GE, Kunz R, Falck-Ytter Y et al. (2008) GRADE: an emerging consensus on rating quality of evidence and strength of recommendations. BMJ 336: 924-926.

6. Pearse RM, Moreno RP, Bauer P, Pelosi P, Metnitz P et al. (2012) Mortality after surgery in Europe: a 7 day cohort study Lancet 380: 1059-1065. 